\title{
TITRE-T-ON SUR LE NET COMME ON TITRE SUR PAPIER - DE L'ÉVOLUTION DU TITRAGE MÉDIATIQUE
}

\begin{abstract}
Jereczek-Lipińska Joanna, Titre-t-on sur le Net comme on titre sur papier - de l'évolution du titrage médiatique [Do we write titles in the Net as we write titles on paper - on the evolution of writing titles in the media]. Studia Romanica Posnaniensia, Adam Mickiewicz University Press, Poznań, vol. XXXII: 2005, pp. 45-55. ISBN 83-232-1465-4, ISSN 0137-2475.

All media are equipped with a specific code of writing bus since always avenement of media causes the appearance of a language even of a technique of writing suitable for the media in question. Each one of these media forges its own technique of communication which continues besides to evolve just like changes its support. We propose to recall this evolution like perceiving the advent of the specific language through one of the elements essential of the media account of the newspaper industry and the cyberpress with knowing titration. It is a challenge by knowing that the internet is not media like the others but a multi-media support, it thus includes all the media in one. Our objective is to define the stage to which the cybertitre in this evolutionary diagram is.
\end{abstract}

Chaque média est doté d'un code d'écriture spécifique car depuis toujours l'avènement d'un média provoque l'apparition d'un langage voire d'une technique d'écriture propre au média en question. Mais avant que ceci arrive véritablement, les journalistes ont d'abord suivi des techniques qui fonctionnaient déjà. Ainsi ils parlaient à la radio comme on écrivait dans la presse écrite, et ils communiquaient à la télévision comme on le faisait à la radio. Ce n'est que bien après, au fur et à mesure du fonctionnement que ces langages ont été adaptés aux supports respectifs. $\mathrm{Au}$ fil du temps chacun de ces médias s'est forgé sa propre technique de communication qui continue d'ailleurs d'évoluer tout comme change son support.

Nous proposons de retracer cette évolution ainsi que percevoir l'avènement du langage spécifique à travers un des éléments essentiels du récit médiatique de la presse écrite et de la cyberpresse (nous écartons dans cette étude les joumaux en ligne qui sont les copies conformes de leur version papier) à savoir le titrage. C'est un défi en sachant que l'Intemet n'est pas un média comme les autres mais un support multimédia, il englobe donc tous les médias en un. Notre objectif est de définir le stade auquel se trouve le cybertitre dans ce schéma évolutif. 


\section{L'(R)ÉVOLUTION DE I_A TITRAILLE DANS LA PRESSE ÉCRITE}

Tout journal tout comme tout article s'accompagnent d'une titraille qui englobe tout titre, surtitre, sous-titre, intertitres, chapeau et/ou mots-clés. Elle désigne une double réalité, d'un côté les appellations de journaux qui apparaissent aux frontispices ou sur la couverture, de l'autre les titrages sur les événements qui organisent l'ensemble de la mise en page. C'est un moment fort du texte, combien de fois, notre lecture commence et s'arrête aux titres.

La façon de titrer dans la presse écrite a considérablement changé dès les titres contenant des informations brutes en passant par le linguistiquement correct pour en arriver au traitement des faits avec des effets de style et des jeux de mots. Nos recherches ont confirmé l'évolution de la forme ainsi que du contenu de la titraille en fonction de différents rôles qu'on accordait à cette partie du langage médiatique. Ainsi nous sommes passés du titrage purement informatif en passant par le vouloir informer, éduquer et impliquer le lecteur en arrivant à la fonction: divertir, séduire, étonner voire même choquer. Le titre est aujourd'hui surtout une sorte de publicité pour le journal ou l'article en question.

\section{LE CHOC DES TITRES - TOUS LES COUPS SONT PERMIS}

Titrer c'est donner une signification à des faits disparates et ceci avec un style, en vue de certains effets pour construire ainsi un discours cohérent répondant à de nombreux impératifs dont la plupart contradictoires. Celui qui s'y lance, construit, déconstruit et reconstruit la langue et comme c'est un journaliste, il invite d'autres à le suivre.

Une des règles d'or de l'écriture joumalistique retentit presque comme une devise française: précision, concision el clarté, nous y rajoutons le plaisir formel obtenu grâce à l'emploi habile du figé. Le titre d'aujourd'hui est imprégné de cette forme verbale. Car le choix des mots dans les titres est loin de relever du hasard. Les mots laissent toujours transpirer un peu plus de sens que celui qu'on leur prête. C'est une véritable institution parce que le sens particulier d'un mot résulte de l'élimination de ceux qu'auraient eus à sa place tous les autres mots admis à paraître dans le même contexte. Il est tout de même vrai que le figé peut être réduit à sa paraphrase mais d'une part il n'est substituable qu'en bloc. D'autre part, même si le figé est paraphrasable, ceci se passe certes, au prix d'appauvrissements sémantiques. La fréquence des structures figées génère des structures moules ou modèles, susceptibles d'une certaine productivité ou plutôt, comme l'atteste notre corpus d'une productivité certaine.

L'écriture journalistique vise à l'économie, dans tous les sens du terme. La tendance générale semble être une réduction graphique de l'énoncé, cc langage médiatique est marqué par le temps et calibré. Le calibrage peut être parfois très 
précis et rigoureusement respecté. Ces impératifs d'espace et de temps ont naturellement une incidence sur l'écriture qui devient un véritable jeu de chiffres et de lettres ainsi que de mots et de sens en l'occurrence le titre se calcule en centimètres voire en nombre de signes. Il s'ensuit que la titraille vise une multiplicité de sens et va donc directement vers la plurivalence du signe et ceci à partir de formes figées le plus souvent ${ }^{\text {. }}$

"Santé ; le casse-tête de la sécu pour tous"

"Bus : à bon port"

l.'Internet dans tous ses éclats

Heureux qui comme Theroux...

"Une comédie légère " plutôt pesante

Le projet de club nautique tombe à l'eau

La langue est l'outil de travail d'un journaliste - titreur de la presse écrite, nous aurions même tendance à dire que c'est le figé qui est l'instrument de son travail. Cet outil est aujourd'hui, plus que jamais avant, multiple surtout que comme l'a très bien dit Véron : « le destinataire d'un type de discours fait partie des conditions de production de ce discours $»^{2}$. Nous observons justement l'évolution vers la complexité à savoir les unités libres s'associent pour former des phrases à structures enchâssées parce que c'est là le moyen de répondre aux pressions d'une communication, qui du fait de l'évolution des rapports sociaux, forme des besoins d'encodage et de décodage de plus en plus complexes.

Par l'emploi et le jeu de la formule figée, le journaliste se veut donner une dimension supplémentaire au récit d'événement car cet élément ćtranger et étrange vient rompre la tranquillité sémantique. Ce journaliste crée et emploie alors des lieux communs étant des lieux ludiques et de reconnaissance.

\section{CES LIEUX COMMUNS QUI DEVIENNENT LIEUX LUDIQUES}

Certes, nous sommes témoins d'un changement, celui qui nous amène à noter le phénomène des jeux de mots dans les titres des récits médiatiques, pas seulement dans une presse célèbre pour ses jeux d'expressions mais de façon généralisée dans les hebdomadaires d'information et même dans des quotidiens nationaux et régionaux.

Le jeu de mots, est loin d'être un procédé linguistique innocent; utilisé volontairement par la presse, la publicité, il n'est ni un amusement, ni un laisseraller : c'est plutôt une intention précise de rompre l'équilibre du langage pour capter

'M.-B. Vittoz Canuto, Si vous avez votre jeu de mots à dire, Analyse de jeux de mots dans la presse et dans la publicité, A.-G. Nizet. Paris 1983.

${ }^{2} \mathrm{M}$. Mathien, Le systeme médiatique; lo journal dans son environnement, in Langue, linguistique, communication - Collection dirigée par Bernard Quemada, Hachette, Paris 1989. 
l'attention. Le bon jeu de mots ne correspond pas à un modèle précis: il naît de l'ambiguïté. Mais l'ambiguîté ne fait-elle pas partie intégrante du langage de tous les jours? Il nous semble d'ailleurs que l'ambiguïté n'est pas une erreur dans le système mais un défi langagier précieux. Et le côté ambigu du langage est la source de sa puissance d'expression, le jeu de mots en est une des manifestations les plus complexes. Mais les jeux ne reposent pas seulement sur des ambiguïtés inhérentes à la langue, ils se fondent également sur l'évocation d'événements ou de citations qu'on sait connus du lecteur.

Le jeu langagier dans la presse constitue tout de même un phénomène relativement nouveau qui s'est imposé, comme l'indique Marie-B. Vittoz Canuto ${ }^{3}$, dans la demière décennie 1970-1980. On pourrait se poser la question sur les raisons de ce renouveau des jeux dans les réseaux de communication. L'une reposerait sur les événements de 1968 qui ont lancé la mode de la libération de l'expression et pendant lesquels on réclamait de «l'imagination au pouvoir ». Ceci a donné naissance à de multiples types de néologismes et parmi eux à des jeux de mots. L'autre viendrait de la «surinformation ». Le nombre incessant d'informations arrivant "en chaîne " de toutes parts fait qu'il faut savoir les mettre en relief dans cette masse communicative. On recourt ainsi à une sorte d'anomalie qui n'est rien d'autre qu'un jeu de mots ayant un rôle séducteur et qui n'a presque plus d'autre effet que celui d'étonner, d'amuser, de séduire. Sa visée est surtout relationnelle, elle cherche donc à accrocher l'attention du lecteur et à l'entraîner dans une relation de complicité. Les jeux de mots ont une valeur esthétique certaine, mais il serait dommage de vouloir réduire son rôle à cette fonction esthétique dont personne ne nie l'importance. Le jeu de langage est bien sûr le jeu de l'esprit. Tout locuteur exerce une activité métalinguistique inconsciente et ceci plus particulièrement dans le domaine du jeu.

Nous allons ici parcourir deux aspects de l'emploi ludique du figé dans les titres, notamment celui de l'analyse des procédés d'une part et celui de l'analyse des effets d'autre part.

Une expression phraséologique, selon Józef Sypnicki ${ }^{4}$, se prête à l'emploi comique mais à condition qu'elle soit entièrement lexicalisée. Il faut se rendre compte du fait que ce sont des jeux de mots qui vérifient en quelque sorte le figement d'une séquence donnée. La lexicalisation des figures peut être considérée comme achevée à partir du moment où le remplacement du mot figuré par un synonyme ou un quasi-synonyme ou autre procédé de modification choque ou fait rire, ce qui constitue justement le ressort de l'humour chez les locuteurs d'une langue.

L'emploi ludique peut être fondé sur la variation formelle, p.ex. Il pleure à froides larmes, elle peut consister dans une opération d'insertion (couper la bonne

${ }^{3}$ M.-B. Vittoz Canuto, Si vous avez votre jeu de mots à dire; Analyse de jeux de mots dans la presse et dans la publicité ; A.-G. Nizet, Paris 1983.

4 J. Sypnicki, Les délexicalisations dans les textes burlesques in Lexique et grammaire, Warszawa 1987. 
parole, reprendre ses saints esprits, saint esprit de contradiction), d'ellipse, de composition, de substitution (ce soir, n'oubliez pas, retrouvez-moi en chair et en voix, vous n'y allez pas de pied mort), d'expansion, de combinatoire, de permutation.

Ou il peut être fondé sur la variation sémantique, elle laisse alors intacte la morphologie de la séquence : Bredouille en rentrant de la chasse : le gibier lui a posé un lapin.

Il y a de différents procédés pour créer un à-peu-près qui est un terme emprunté à Michaela Heinz ${ }^{5}$ et recouvre justement des déformations d'une locution de base. Mais l'APP n'est pas toujours issue d'un jeu de mots, d'un jeu prémédité sur le sens des mots. Ainsi l'APP, au sens plus global, peut également être le résultat d'un lapsus, par exemple : revenons à nos boutons, ça ne fait pas oncle d'un doute!, noblisse oblège!

Et puis on ne peut pas tout savoir, il y a donc des jeux de mots issus de l'ignorance. L'étymologie populaire repose sur la paronymie, le locuteur remplace alors un mot qu'il ne connaît pas, ou qu'il a mal compris, par un paronyme plus clair pour lui. En voici un exemple ${ }^{6}$ :

Ronsard a écrit un poème où il explique que la rose perd les pédales en l'espace d'un matin, Rassure-toi, ma chère maman, je suis à la pension comme un vrai coq en plâtre.

Il s'ensuit que les APP peuvent avoir des origines différentes. Ce qui leur est commun, par contre, c'est le fait qu'ils sont d'abord occasionnels, individuels, relevant du discours. A force d'être diffusé et répété, un APP peut se lexicaliser et être employé et ceci pour plusieurs raisons, notamment par plaisanterie, par exemple celle que Coluche a rendu célèbre, partir, c'est crever un pneu.

La créativité quasi automatique qui s'exprime dans le mot d'esprit semble éloigner absolument celui-ci du calcul : l'auteur d'un bon mot ne cherche pas, il trouve. D'où l'existence des journalistes ou même des journaux qui fleurissent de jeux de mots à côté de ceux qui n'en ont guère.

Ce sont des journaux pas comme les autres qui témoignent des possibilités que donne la langue figée à ceux qui savent la jouer, déjouer et rejouer. Au sein de ces titres il y a lieu d'insister sur un rôle provocateur de la langue, c'est une arme dont la puissance est mesurée en fonction des cibles visées voire touchées. Il faut souligner ici que dans la communication des médias tels que nous les avons vus, le message doit passer; c'est pourquoi l'allusion est le plus souvent transparente, évidente perdant ainsi de son intérêt; il n'en va pas toujours de la sorte lorsque l'allusion correspond à des intentions satiriques.

\footnotetext{
${ }^{5} \mathrm{M}$. Heinz, L’à-peu-près dans les locutions et son traitement lexicographique, in P. Fiala, P. La fon, M.-P. Piquet, La locution : entre lexique, syntaxe et pragmatique, Klinksieck, Paris 1997.

${ }^{6}$ Les exemples de la Foire aux cancres de Jean-Charles tirés de ... cf. le titre ci-dessus.
} 
En tout cas, jouant avec et sur les mots, en usant de la langue selon un mode qui lui est propre, le journal éclaire, transforme, commente, organise l'information. Les expressions figées sont omniprésentes au sein de la presse avec une priorité notoire pour toute sorte de titres, il n'y a donc pas de lieux interdits.

\section{DES LIEUX LUDIQUES AUX LIEUX DE RECONNAISSANCE}

Il y a plusieurs niveaux de lecture d'un récit médiatique et en ce qui conceme les titres les deux premiers nous intéresse particulièrement. La première étape est le survol des titres autrement dit le feuilletage et ce n'est que dans le deuxième niveau de lecture que nous découvrons le chapeau, les attaques et les encadrés. Vient après une éventuclle lecture linéaire. Ces lectures sont encore déterminées par le contenu à savoir notre intérêt dépend des lois de proximité, en fonction de la distance géographique et temporaire de l'information, nous poursuivons ou pas la lecture. Notamment nous sommes plus sensibles à ce qui se passe plus près de chez nous et dans l'immédiat. Mais l'habillage de ce contenu est tout aussi important à savoir nous sommes beaucoup plus tentés par un bon jeu d'esprit que par un message sec et plat. Ceci présuppose un autre niveau de connivence dans la lecture entre celui qui émet ce message et celui qui le décode.

Effectivement le figement n'est pas seulement un phénomène intrinsèque mais relève d'un jugement porté par les locuteurs sur certaines séquences en discours. Pour qu'on puisse parler de figé, il est indispensable que l'on soit à même de porter sur un segment un jugement double : synthétique d'une part, par lequel la locution est assimilée par synonymie à un mot unique; analytique d'autre part, par lequel la locution est envisagée comme un syntagme ordinaire dont la valeur résulte plus ou moins de la composition des valeurs respectives des composants. L'effet locutionnel résulte alors de la tension voire «miroitement", comme l'indique Pêcheux", entre les effets de sens des deux analyses. Cette dualité d'analyse présuppose trois dimensions abordées déjà par Pierre Achard et Pierre Fiala ${ }^{8}$ - la première serait une dimension linguistique interne liée à la morphosyntaxe. La seconde relèverait de l'identité sociologique du locuteur et enfin la dernière serait liée à l'activité métadiscursive - une dimension stylistique. Mais le jugement de locutionnalité implique aussi la compétence grammaticale car le locuteur donne en même temps son jugement de grammaticalité de l'énoncé. Et enfin l'intuition locutionnelle appartient également à la compétence communicative. Autrement dit jouer et pouvoir comprendre relève de la compétence désirée mais pas donnée à tout le monde.

${ }^{7}$ M. Pêcheux, Matéricl en vuc de l'article Complétive/hfinitifs/Infinitives. Linx 10, 1984, p. $7-22$.

${ }^{8} \mathrm{P}$. Achard, P. Fiala, La locutionnalité à géométrie variable, in P. Fiala, P. Lafon, M.-F. Piquet, La locution : entre lexique, syntaxe et pragmatique, Institut National de la languc française, Klinksieck, Paris 1997. 
Bref, comme nous venons de le démontrer le titre, dans la presse écrite traditionnelle dans cet espace de plus en plus contraignant, est de plus en plus complexe, voilé, truffé d'expressions. C'est aujourd'hui plus que jamais avant un véritable jeu de chiffres et de lettres ainsi que de mots et d'expressions et comme l'annonce le slogan publicitaire du Monde - Tout y est dit, à vous de voir !

Il nous reste à confronter cette évolution du titre de la presse écrite avec celle du titre sur le Web ce qui permettra de tracer les pistes d'avenir de ce denier.

\section{L'IMMÉDIATETÉ OBLIGE - CES CONVERGENCES DU CADRE DE L'ÉNONCIATION QUI DIVERGENT BEAUCOUP}

La presse écrite traditionnelle ne fonctionne plus à sens unique comme l'indique Bourdieu", les récits médiatiques sont des « ocuvres à peu près exclusivement déterminces et dominées par la représentation des attentes du public ». Tout de même sur l'Internet nous avons affaire à un phénomène nouveau dans ce domaine à savoir l'interactivité qui réduit considérablement le statut du journaliste en lui attribuant un autre rôle, il n'est désormais qu'une interface dans la communication médiatique. Tout ceci au profit du cyberlecteur qui, lui fait désormais partie du média, notamment il peut donner sa réaction ou converser directement aussi bien avec d'autres internautes qu'avec le scripteur par le biais de "chats", de forums de discussion et de sondages. Cette possibilité d'échange immédiat est un des atouts considérable du Net.

En outre, la cyberécriture tout comme l'écrit sur papier doit suivre un certain nombre d'impératifs encore une fois bien souvent contradictoires. Les textes sont également calibrés, le titreur a un espace et un temps bien déterminés même si les possibilités techniques témoignent du contraire. En tout cas, la lisibilité, la visibilité du site ainsi que l'urgence du message exigent les contraintes évidentes à ce niveaulà, tout comme elles appellent à une structuration rigoureuse et sans faille.

L'e-titre reprend la fonction de base de la titraille classique à savoir son rôle principal serail d'informer l'internaute mais tout aussi le guider à travers le réseau.

\section{L'E-TITRE - LES CARACTÉRISTIQUES DU GENRE : LA RIGUEUR EST DE RIGUEUR}

Comme les informations sur la toile arrivent en continu et sont donc sans cesse renouvelées et réactualisées, les messages sont denses et souvent illustrés par d'autres médias (document iconique, audio, vidéo) le risque est de trop charger la page en informations, on pourrait alors trouver le site illisible et le quitter. Les etitres sont censés nous aider à nous repérer dans cette masse communicative.

${ }^{9}$ P. Bourdicu, Champ intellectuel et projet créateur, Temps modernes, 1966, $1^{\circ} 246$, pp. 874-875. 
Leurs caractéristiques principales c'est d'être extrêmement concis, précis et exacts. Un simple coup d'œil devrait suffire au cyberlecteur pour constater s'il est sur la bonne page. Ces titres lui diront où il se trouve exactement comme s'il lançait une recherche par les moteurs de recherches.

En outre, la devise d'un e-titre est la même que pour le journal papier à savoir il s'agit d'exprimer le maximum d'informations en minimum de mots. Mais cette devise s'applique à deux cadres divergents en l'occurrence c'est l'internaute qui dirige sa lecture, il en est maître absolu. Evoquons encore une fois la notion des niveaux de lecture et des lois de proximité car ils sont encore plus manifestes ici. Le premier niveau de la cyberlecture présuppose un parcours des yeux en diagonal de la page et non linéaire comme c'est le cas de la presse papier. L'internaute «scanne » la page d'où la nécessité de bien mettre en relief tous les e-titres, intertitres, mots-clé. Au second niveau il parcourt le chapeau et les encadrés. Ceci présuppose un système différent d'organisation des données.

La page d'accueil d'un site est déterminante, après avoir traversé les deux niveaux de lecture il faudrait que l'internaute ait tous les éléments nécessaires pour décider s'il poursuit sa lecture ou pas. Vu les frais éventuels ou la vitesse de connexion, le cyberlecteur aime pouvoir vite évaluer la pertinence d'un texte. La titraille doit ainsi informer c'est à dire contenir des réponses aux maximum de questions et ceci en parfaite structuration - Où ? Quand ? Qui ? Quoi ? Comment Pourquoi ? Avec quelles conséquences? (ce qui dans la presse écrite se trouve dans le corps de l'article - l'effet de la pyramide inversée). On pourrait comparer ces cybertitres de la page d'accueil à la recette de cuisine : Poulet à la bière d'abbaye façon carbonade. Derrière l'intitulé se trouve les ingrédients principaux ainsi que la préparation. Si le plat nous met de l'eau à la bouche et les produits sont accessibles, on lira la suite. Les cyberécritures utilisent ces procédés en visant l'efficacité maximale, en voici un exemple :

Page d'accueil du journaldunet.com du 6 janvier 2005

Mots clés : Actualité - Bourse

Titre : Première séance de l'année à la baisse pour le CAC 40

Les lois de proximité sont tout de suite données:

Proximité géographique : Le CAC 40 induit la France

Proximité temporelle : actualité - la première séance de l'année

Le titre ainsi que le chapeau qui suit, résument l'essentiel de l'information en répondant aux questions que l'on se pose :

Quoi : la baisse - la bourse de Paris recule de 0,88

Qui : Total, Sanofi, les technologies.

L'ensemble : titre de rubrique - titre - chapeau - intertitres est une sorte de «vitrine ${ }^{10}$ informative et indicative. C'est sobre, ça va droit au but, il n'y a pas

${ }^{10}$ A. Dejond, La cyberlangue française, la renaissance du livre, Tournai 2002, p. 84. 
d'effet de style ni de jeux de mots. Afin d'obtenir une efficacité et une lisibilité optimales les e-titres sont transparents, clairs et dynamiques. Et pour ceci on écarte tout mot abstrait ou mot-outil, on évite les adjectifs et les verbes vides de sens (p.ex être). On emploie seulement les mots pleins comme p.ex. on opte plutôt pour «Hôpital en feu : 5 morts » que «Un hôpital est en feu et provoque la mort de 5 personnes». Ainsi pas de mots-outils (articles, prépostions), pas de verbes («est», «provoque »), cinq mots pleins au lieu de douze qui transmettent bien le message.

L'effet dynamique de l'e-titre s'obtient par le fait qu'on évite la redondance et on préfère la voix active à la voix passive.

Tout titre se veut précis et pertinent à savoir toute information est censée apporter du nouveau. On ne lira pas en ligne que « Le cancer tue » mais plutôt « Le cancer a tué 2431 fois cette année ».

Le chapeau a un autre rôle important à jouer. Il donne un résumé de l'article, dessine sa structure : Le journaldunet.com du 6 janvier 2005 : "Amar Lakel, chercheur en sciences de l'information et de la communication, dresse un portrait des bons et des mauvais élèves de l'e-administration, et en précise les enjeux ».

C'est tout un petit texte mais dont les phrases sont simples, courtes ou avec les subordonnées en tête et l'apposition plutôt que les parenthèses.

Nous avons également souvent affaire au plan cinématographique qui fonctionne par séquences autonomes, tout paragraphe est indépendant et traite exhaustivement un autre aspect. L'intertitre joue alors le rôle du titre, ainsi à la mort de George Ilarrison lalibre.be a proposé dans le chapeau les trois parties de l'article :

"L'importance musicale des Beatles pour le vingtième siècle.

Le rôle de George Harrison au sein du groupe,

Sa carrière chaotique en solo $" 1$.

Nous pouvons ici lire chacune de ces parties indépendamment ou toutes les parties ensemble, libre à nous de gérer notre lecture, ce qui dans la presse écrite traditionnelle est imposé d'emblée.

La toile implique une autre façon d'informer, une structure et une écriture différentes. Une fois encore, le français s'adapte au support.

\section{LE JEU CONTINUE...}

Il est sûr et certain que l'on ne titre pas sur le net comme on titre dans la presse écrite mais le cybertitre d'aujourd'hui est en passe de forger son langage. On pourrait se poser la question de savoir si on a vraiment besoin de ce code d'écriture spécifique mais la réponse ici s'impose pour au moins une raison - on est déjà passés par l'étape de la mise des journaux écrits tels quels en ligne. L'entreprise n'a pas vraiment rencontré beaucoup de succès car on passait à côté des possibilités

${ }^{11}$ Ibidem, p. 86. 
techniques offertes par ce média. C'est là où la culture en ligne a fait son apparition et son principe de départ a été de titrer ou plus globalement d'informer autrement.

Ce langage n'est pas aussi marqué que le langage des «chatteurs " avec ses nombreuses abréviations, sigles, anglicismes, émoticônes. C'est la preuve flagrante qu'il se trouve seulement dans la phase intermédiaire entre le style «télégraphique » du début de la presse, son adaptation au support multimédia et la naissance du nouveau langage. Il faudrait ici prendre en considération le fait que si on n'exploite pas encore, à nos yeux, un véritable potentiel de l'Internet c'est tout aussi parce que les journalistes ne sont pas formés à cet égard. En effet, il y a très peu de cours de journalisme spécifiques à l'Internet (nous en avons recensé quelques uns aux EtatsUnis).

Certes, une évolution est en cours, celle qui nous amène quand même à noter le phénomène des jeux de mots dans les e-titres ce qui n'était pas encore le cas il y a très peu et ce qui prouve que l'on a vite réalisé que si le jeu de mots et d'expressions emprunte les détours du langage, c'est tout aussi pour mieux prendre les raccourcis sémantiques. Et puis, cette face cachée métaphorique joue le rôle d'un véritable signal parce qu'il éveille l'attention du lecteur, en voici un échantillon :

"OpelRent.ji souhaite passer à la vitesse supérieure" - journaldunet.com du 6 janvier 2005 ;

"Clics d'Or 2005 : climat au beau fixe »- journaldunet.com du 5 janvier 2005 ;

"Comparatif: les mails gratuits au banc d'essai » - l'internaute du 6 janvier 2005

A quand ce langage propre à la presse en ligne car entre ce jeu incessant et provocateur dans les titres de la presse écrite et la rigueur encore assez absolue des e-titres il y a encore un grand espace à aménager.

\section{BIBLIOGRAPHIE}

Achard P., Fiala P. (1997), La locutionnalité à géométrie variable, in P. Fiala, P. Lafon, M..P. Piquet, La locution : entre lexique, syntaxe et pragmatique, Institut National de la langue française, Klinksicck, Paris.

A nis J. (1998), Texte ét ordinateur, l'écriture réinventée, De Boeck.

Bourdieu P. (1966), Champ intellectuel et projet créateur, Temps modernes, $\mathrm{n}^{\circ} 246$, pp. 874-875.

Breton P. (2000), Le Culle de l'Internet, La Découverte.

Dejond A. (2002), La cyberlangue française, La renaissance du livre, Tournai.

Duneton C., Pagliano J.P. (1978), Anti-manuel de français, Ed. du Seuil. Paris.

Gross G. (1996), Les expressions figées en français, noms composés et autres locutions, Ophrys Paris. Guerin S. (1996), La cyberpresse, Hermès.

Heinz M. (1997), L’à-peu-près dans les locutions et son traitement lexicographique, in P. Fiala, P. La fon, M.-P. Piquet, La locution : entre lexique, syntaxe et pragmatique, Institut National de la langue française, Klinksieck, Paris. 
Labasse B. (1999), La lisibilité rédactionnelle; fondements et perspectives, Communication et Langages.

Lapointe P., Le journalisme à l'heure du Net, Les Presses de l'Universitć.

Mathien M. (1989), Le système médiatique ; le journal dans son environnement, in Langue, linguistique, communication - Collection dirigée par Bernard Quemada, Hachette, Paris.

Pêcheux M. (1984), Matériel en vue de l'article Complétive/Infinitifs/Infinitives, Linx 10, p. 7-22.

Sypnicki J. (1987), Les délexicalisations dans les textes burlesques, in Lexique et grammaire, Warszawa.

Vittoz Canuto M.B. (1983), Si vous avez votre jeu de mots à dire; Analyse de jeux de mots dans la presse et dans la publicité; A.-G. Nizet, Paris.

Yaguello M. (1981), Alice au pays du langage. Pour comprendre la linguistique, Ed. de Seuil, Paris.

http://www.linteranutc.com

hitp://www.journaldunet.com

hıtp://www.lalibre.be 